\title{
Complex finite element analysis applied to photothermal and thermoelastic microscopies
}

\author{
B. Cavallier ${ }^{\text {a }, ~ S . ~ B a l l a n d r a s, ~ B . ~ C r e t i n ~ a n d ~ P . ~ V a i r a c ~}$ \\ Institut FEMTO-ST, Dept. LPMO UMR 6174 CNRS-Université de Franche-Comté, 32 av. de l'Observatoire, \\ 25044 Besançon Cedex, France
}

Received 12 November 2007, accepted 4 February 2008

\begin{abstract}
Photothermal and thermoelastic microscopes are non-destructive systems that generally work with lock-in detection. Experimental results are typically given for one modulation frequency. In order to understand related magnitude and phase images obtained with complex geometries, we have developed a finite element analysis (FEA) dynamic method. Particularly, it enables to directly obtain both thermal and thermoelastic harmonic fields for one modulation frequency. It has been applied to thermoelastic microscopy and has shown very good agreement with experiments. Numerical results from a 3D complex geometry model are presented and show the influence of both excitation size radius and modulation frequency on thermoelastic normal displacements.
\end{abstract}

\section{Introduction}

Solids photothermally excited by a focused laser beam have been fully described by many theoretical models $[1,11,14,19]$. Related analytical models have been developed in order to describe the generated thermal field $[7,11,13,14]$. The heat source generated by laser irradiation induces elastic deformations that yield surface displacement recordable by a laser probe. With this approach, the corresponding thermoelastic response can be described by using the thermoelastic wave equations [16]. Much work has been done regarding the resultant thermoelastic displacement field. Most of it concern threedimensional analytical models $[6,12,15,18]$. Some papers also consider the dynamic case [10], however these studies are restricted to simple geometries.

Finite element analysis of photothermally excited structures by an intensity modulated laser has been studied by only a few authors $[8,20]$. Subsequently we have focused on finite element methods [21] and particularly in Modulef [9] to theoretically study the responses of $2 \mathrm{D}$ or 3D general structures. Many moduli devoted to piezoelectric harmonic responses have been developed, based on the Modulef FEA toolbox [9], at FEMTO-ST (LPMO dept.) [2]. One of the corresponding code enables to simulate the linear (small displacement assumption) piezoelectric response of $2 \mathrm{D}$ or $3 \mathrm{D}$ structures submitted to harmonic excitation. It should be noted that thanks to the complex analysis, the material intrinsic losses are also taken into account. Then we have done two complex analysis moduli for thermal simulations and for thermoelastic coupling. The mechanical stresses could also be obtained with the native tools of Modulef.

\footnotetext{
${ }^{a}$ Corresponding author: bruno.cavallier@femto-st.fr
}

In this paper we will investigate this FEA dynamic method. Based upon complex analysis for one modulation frequency, this method particularly appears to be well suited for models that require frequencies greater than $100 \mathrm{kHz}$. First part of this paper describes the FEA dynamic method that has been implemented using the opened FEA toolbox Modulef, first developed by INRIA Rocquencourt [9], for both thermal and thermoelastic problems. A preliminary step has consisted in testing and validating the coupled thermoelastic FEA on a complex 3D geometry with anisotropic material properties. In these experiments an etched silicon wafer was scanned on its opposite face by an argon ion laser for photothermal excitation and by a laser probe to detect both photothermal and thermoelastic fields. This validation has been established in a previous paper [5] so that we are able to simulate thermal and thermoelastic images (magnitude and phase). The second part then shows theoretical results of both radius excitation size and modulation frequencies influence on this sample.

\section{Theory: complex FEA}

\subsection{Thermal problem}

The partial derivative equations form of the thermal dynamic problem is given below.

$$
\left\{\begin{array}{l}
\text { Find } T(x, y, z) \text { complex temperature such as: } \\
\rho c \frac{\partial T}{\partial t}-\left(\kappa_{i j} T_{, j}\right)_{, i}=q^{\Omega} \text { that is to say } \\
\quad j \Omega \rho T-\left(\kappa_{i j} T_{, j}\right)_{, i}=q^{\Omega} \text { in } \Omega \\
\phi=\left(\kappa_{i j} T_{, j}\right) n_{i} \text { in } \Omega \\
\phi+g T=q^{\Gamma} \text { over } \Gamma_{0} \\
T=\bar{T} \text { over } \Gamma_{1}
\end{array}\right.
$$


where $\rho$ is the material mass density, $c$ is the material specific heat, $\kappa_{i j}$ is the thermal conductivity tensor, $T,{ }_{j}$ is the first partial derivative of $T$ function versus $x_{i}, \phi$ is the heat flux, $n_{i}$ is the $i$-th component of the outwards oriented normal to $\Gamma_{0}, g$ is the heat convection coefficient through $\Gamma_{0}$ boundary, $\bar{T}$ is the complex constrained temperature over $\Gamma_{1}$, and $X_{i i}=\sum_{i} X_{i i}$ as Einstein indices convention is used.

We should note that the whole thermoelastic coupling is not treated here (i.e. elastic $\rightarrow$ thermal and thermal $\rightarrow$ elastic). Indeed it has been shown [3] that photothermal heat sources are $10^{7}$ to $10^{10}$ greater than coupling elastic heat sources for the thermoelastic microscopy considered problem. Thus, we will only consider the thermoelastic coupling thermal $\rightarrow$ elastic. As a consequence, the general heat conduction equation [16]:

$$
\rho c \frac{\partial T}{\partial t}-\left(\kappa_{i j} T_{, j}\right)_{, i}+\eta \frac{\partial\left(U_{k, k}\right)}{\partial t}=q^{\Omega}
$$

where $U_{i}(x, y, z)$ is the $\mathrm{i}$-th component of the complex displacement field at $M(x, y, z)$ point and $\eta$ is the elastic $\rightarrow$ thermal coupling coefficient, is reduced to:

$$
\rho c \frac{\partial T}{\partial t}-\left(\kappa_{i j} T_{, j}\right)_{, i}=q^{\Omega}
$$

which brings us back to equation (1).

We can then write the strong Galerkin integral form related to the dynamic thermal problem:

$$
\int_{\Omega} \delta T\left(\rho c \frac{\partial T}{\partial t}-\left(\kappa_{i j} T_{, j}\right)_{, i}\right) d \Omega=\int_{\Omega} \delta T q^{\Omega} d \Omega .
$$

By adopting the convention $T_{, j}=\frac{\partial T}{\partial a_{j}}$ and applying the Green-Ostrogradsky transform to the heat flux term in the Galerkin integral in order to write the weak integral form:

$$
\begin{aligned}
\int_{\Omega} \delta T \frac{\partial\left(\kappa_{i j} T_{, j}\right)}{\partial a_{i}} d \Omega=\oint_{\Gamma} \delta T\left(\kappa_{i j} T_{, j}\right) n_{i} d \Gamma \\
\quad-\int_{\Omega} \frac{\partial \delta T}{\partial a_{i}}\left(\kappa_{i j} T_{, j}\right) d \Omega .
\end{aligned}
$$

Finally, if we take into account the harmonic state assumption (at angular frequency $\Omega$ ) then the Galerkin weak integral form can be written as:

$$
\underbrace{\underbrace{}_{\text {Seat density source }}\left(\frac{\partial \delta T}{\partial a_{i}} \kappa_{i j} \frac{\partial T}{\partial a_{j}}+\delta T j \Omega \rho c T\right) d \Omega+\oint_{\Gamma_{0}} \delta T g T d \Gamma}_{([K]+j \Omega[M])}=
$$

The discretization of equation (6) yields to the final thermal matrix system. It is described by the stiffness matrix
$[K]$, the mass matrix $[M]$, the temperature vector $\{T\}$ and the thermal excitation vector $\{Q\}$.

$$
\Longrightarrow([K]+j \Omega[M])\{T\}=\{Q\} .
$$

This system can be solved by many methods. We have chosen a Cholesky method [17] since our matrices exhibit positive definite properties.

\subsection{Thermoelastic problem}

The dynamic thermoelastic problem as partial derivative equations form is given below.

$$
\left\{\begin{array}{c}
\begin{array}{rl}
\text { Find } U_{i}(x, y, z) & \text { complex displacement field } \\
\text { such as: }
\end{array} \\
\begin{array}{rl}
\rho \frac{\partial^{2} U_{i}}{\partial t^{2}}-\sigma_{i j, j}=f_{i}^{\Omega} \text { that is to say } \\
\quad-\rho \Omega^{2} U_{i}-\sigma_{i j, j}=f_{i}^{\Omega} \text { in } \Omega
\end{array} \\
\sigma_{i j} n_{j}=f_{i}^{\Gamma} \text { over } \Gamma_{0} \\
U_{i}=\overline{U_{i}} \text { over } \Gamma_{1}
\end{array}\right.
$$

where $\sigma_{i j}$ is the stress tensor (the stress tensor is linked to the strain sensor by material behaviour law).

As the thermoelastic coupling thermal $\rightarrow$ elastic has only to be considered in our case, the following thermoelastic behavior laws are injected in equation (8):

$$
\sigma_{i j}=C_{i j k l}\left[\varepsilon_{k l}-\varepsilon_{k l}^{T}\right]=C_{i j k l}\left[\varepsilon_{k l}-\alpha_{k l}\left(T-T_{0}\right)\right]
$$

where $C_{i j k l}$ is the elasticity tensor, $\varepsilon_{k l}$ is the linearized and symmetrized strain tensor (Green-Lagrange quasistatic assumptions): $\varepsilon_{i j}=\frac{1}{2}\left(U_{i, j}+U_{j, i}\right), \varepsilon_{k l}^{T}$ is the symmetric thermal strain tensor, $\alpha_{k l}$ is the thermal expansion tensor, and $T_{0}$ is the temperature field for $M(x, y, z)$ point at time $t=0$.

The previous relation describes a part of the thermoelastic coupling and directly acts in the dynamic thermoelastic behavior. Starting from equation (8), we can write the Galerkin strong integral form related to the dynamic thermoelastic problem:

$$
\int_{\Omega} \delta U_{i}\left(\rho \frac{\partial^{2} U_{i}}{\partial t^{2}}-\sigma_{i j, j}\right) d \Omega=\int_{\Omega} \delta U_{i} f_{i}^{\Omega} d \Omega .
$$

By adopting the convention $\sigma_{i j, j}=\frac{\partial \sigma_{i j}}{\partial a_{j}}$, we apply the Green-Ostrogradsky transform to the stress tensor in the Galerkin integral:

$$
\begin{aligned}
\int_{\Omega} \delta U_{i} & \frac{\partial \sigma_{i j}}{\partial a_{j}} d \Omega \\
& =\int_{\Omega} \delta U_{i} C_{i j k l}\left(\frac{\partial^{2} U_{l}}{\partial a_{j} \partial a_{k}}-\alpha_{k l} \frac{\partial T}{\partial a_{j}}\right) d \Omega \\
& =\oint_{\Gamma} \delta U_{i} \sigma_{i j} n_{j} d \Gamma-\int_{\Omega} \frac{\partial \delta U_{i}}{\partial a_{j}} \sigma_{i j} d \Omega .
\end{aligned}
$$


As far as the thermoelastic fields are assumed to be harmonic (at angular frequency $\Omega$ ), the Galerkin weak integral form can be finally written as (details of the calculations are given in Ref. [5]):

$$
\begin{aligned}
& \underbrace{\int_{\Omega}\left(\delta S_{i j} C_{i j k l} S_{k l}-\delta U_{i} \rho \Omega^{2} U_{i}\right) d \Omega}_{\left([K]-\Omega^{2}[M]\right)}= \\
& \underbrace{\int_{\Omega}^{\Omega} \delta S_{i j} C_{i j k l} \alpha_{k l} T d \Omega}_{\text {Thermoelastic coupling }}+\underbrace{\int_{\Omega} \delta U_{i} f_{i}^{\Omega} d \Omega}_{\text {Volumic force }}+\underbrace{\oint_{\Gamma_{0}} \delta U_{i} f_{i}^{\Gamma} d \Gamma}_{\text {Surfacic force }} .
\end{aligned}
$$

The discretization of equation (12) yields to the final thermoelastic matrix system. It is then described by the stiffness matrix $[K]$, the mass matrix $[M]$, the displacement vector $\{U\}$ and the excitation vector $\left\{F_{\text {thermal }}+F\right\}$. Then a direct method is used to solve the final thermoelastic matrix system.

$$
\Longrightarrow\left([K]-\Omega^{2}[M]\right)\{U\}=\left\{F_{\text {thermal }}+F\right\} .
$$

\section{Numerical FEA results}

So far we have described the FEA and the calculations applied to thermal and thermoelastic dynamic problems. Now we will apply a focused laser beam as thermal boundary conditions. We have followed reference [11] for describing the photothermal source. Typically it is a focused laser beam with an intensity distribution that follows a gaussian law corresponding to a $T E M_{00}$ mode. More precisely, the heat flux density modulus $J(x, y)$ is in our case:

$$
\begin{aligned}
|J(x, y)|=\frac{P_{\text {laser }}(1-R)}{\pi w^{2}} & \\
& \times \exp \left(\frac{-\left(x-x_{L}\right)^{2}-\left(y-y_{L}\right)^{2}}{w^{2}}\right) .
\end{aligned}
$$

The notations of equation (14) are explained in Figure 1. $P_{\text {laser }}$ is the total power of the excitation laser, $R$ is the sample reflectivity, $w$ is the $1 / \mathrm{e}$ intensity radius of the excitation beam and $\left(x_{L}, y_{L}\right)$ are the coordinates of the excitation laser position. Particularly, this figure shows the 3D modeling strategy of the laser sweep.

Figure 2 shows the mesh. Particularly, we have taken care of the spatial sampling for the propagation of thermal and elastic waves and for the modelling of the photothermal gaussian excitation source. The silicon sample has been meshed in a relevant way. Practically, the mesh has been refined close to the impact point of the heat flux. According to the Shannon theorem at least two samples (the nodal values of the unknown fields) are required for the given characteristic length of the excitation source to provide pertinent information about its spatial contribution. Consequently, the mesh must be refined close to the impact point of the photothermal source in order to correctly take into account the localized excitation (Fig. 2).
In our case, the characteristic length of the excitation source is given by the $1 / e$ intensity radius of the excitation beam $w$. As a result of the Shannon theorem, in that region the finite element size must be less than $w / 2$. This condition is fully satisfied as $w / 2$ is about $1.1 \mu \mathrm{m}$ and the smallest element size in that region is below $0.1 \mu \mathrm{m}$ with 4 elements along $1.1 \mu \mathrm{m}$. This figure shows a part of the elementary mesh (block of $20 \times 40 \times 10 \mu \mathrm{m}^{3}$ ) that will be connected to more general mesh.

The accuracy analysis of the FEA thermoelastic model has been fully described in reference [4]. The polynomial interpolation degree, the mesh density and the efficiency of the algorithm used to solve the final linear system [21] govern the accuracy of the FEA results.

For imaging applications and image processing techniques, normalized results are typically used. Thus, Figure 3 gives an example of the normalized dynamic thermoelastic magnitude for a $500 \mu \mathrm{m}$ sweep of the silicon sample. The considered experimental parameters are $w=$ $0.5 \mu \mathrm{m}$ and $w=5 \mu \mathrm{m}$ for $10 \mathrm{kHz}, 100 \mathrm{kHz}$ and $1 \mathrm{MHz}$ modulation frequencies. This figure clearly shows that the thermoelastic displacement results depend firstly on the the size of the excitation source and secondly on the modulation frequency.

Let us develop the previous claims with a qualitative analysis. As we can see on these figures, the dynamic range is better for the $5 \mu \mathrm{m}$ excitation size radius than for the $0.5 \mu \mathrm{m}$ case. This phenomenon can be easily explained with an analogy of thermal fields. It is proven that thermal diffusion length depends on the modulation frequency as the thermal source is greater than the thermal wavelength. But in thermal near fields (i.e. the thermal excitation source is smaller than the thermal wavelength which is typically our case) both thermal penetration depth and thermal lateral resolution mainly depend on the excitation size radius and the frequency dependence becomes negligible.

On one hand the subsurface structure to detect in the etched silicon sample is located $30 \mu \mathrm{m}$ below the surface, and on the other hand the thermal energy is deeper for the $5 \mu \mathrm{m}$ excitation size radius than for the $0.5 \mu \mathrm{m}$ case. Thus, for $w=5 \mu \mathrm{m}$ the related thermoelastic displacement fields are more sensitive to the substructure to detect than for the other case which explains the dynamic range difference.

As concerns the modulation frequency dependence, the interpretation must be given for each excitation size case. For the first case (i.e. $w=0.5 \mu \mathrm{m}$ ) the near field effects are significant so that the thermal energy is concentrated near the surface (about one $\mu \mathrm{m}$ ). Moreover the higher is the modulation frequency the lower is the thermal penetration depth. As a result the lowest modulation frequency $(10 \mathrm{kHz})$ shows a better dynamic range. But for the $w=5 \mu \mathrm{m}$ case, the differents thermal diffusion length as a one dimensional approximation are respectively equal to $54 \mu \mathrm{m}$ for $10 \mathrm{kHz}, 17 \mu \mathrm{m}$ for $100 \mathrm{kHz}$ and $5 \mu \mathrm{m}$ for $1 \mathrm{MHz}$. Hence for the $10 \mathrm{kHz}$ modulation frequency the subsurface structure is easily detected but the signal is averaged as the thermal diffusion length 

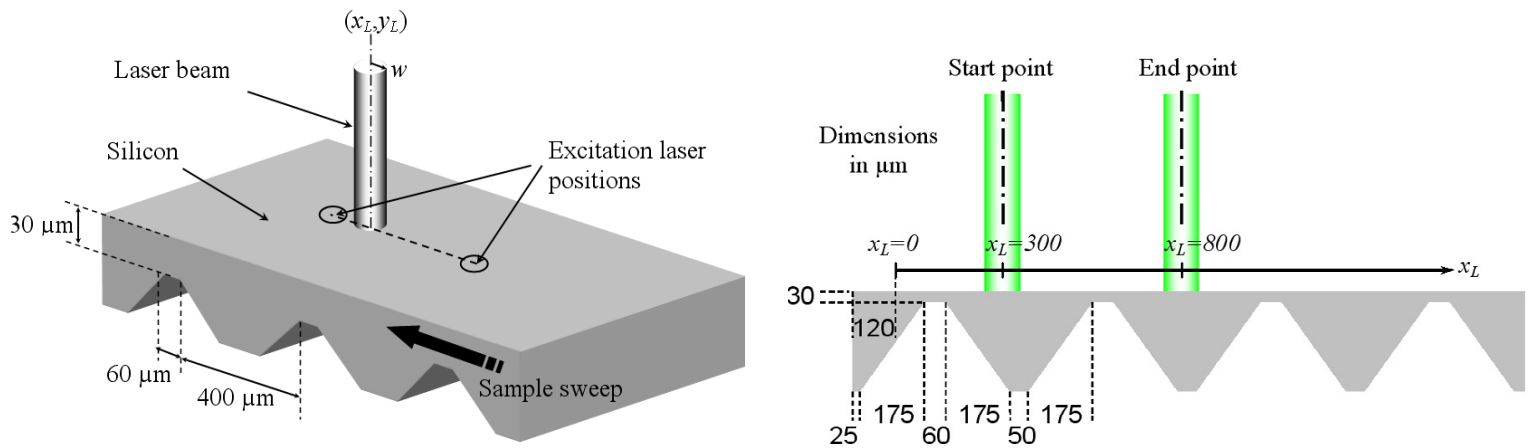

Fig. 1. Geometry of the silicon sample and 3D modeling strategy.

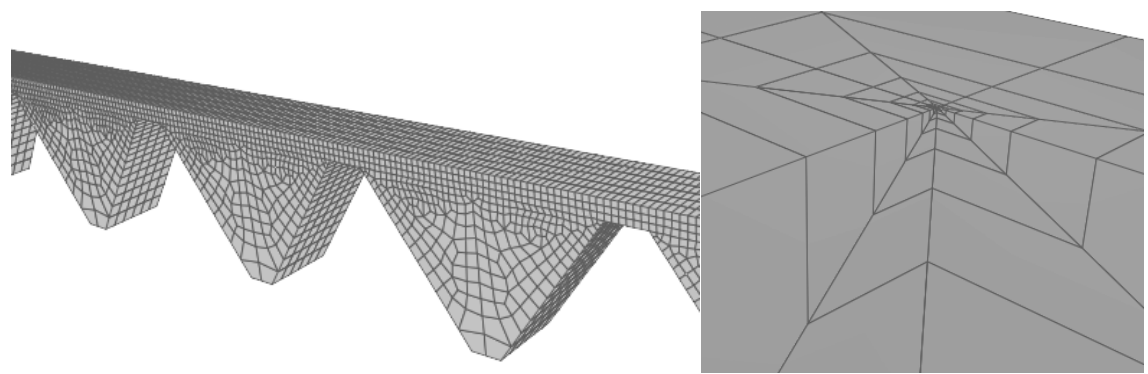

Fig. 2. Sample mesh and refined part of the mesh (meshing of the photothermal impact area).
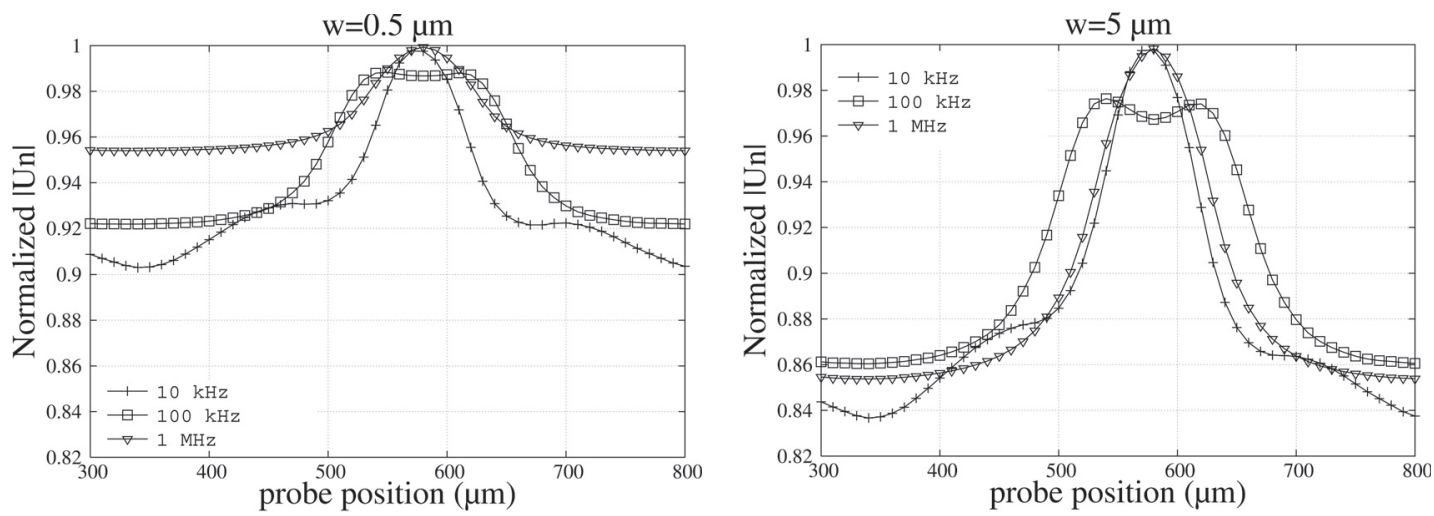

Fig. 3. Normalized thermoelastic displacement magnitude.

overcomes the substructure. For the $1 \mathrm{MHz}$ modulation frequency, the thermal energy is not enough deep to correctly detect the substructure. Finally, the $100 \mathrm{kHz}$ modulation frequency yields to a thermal diffusion length that is in the same order of the substructure depth so that thermoelastic displacement gives more informations on the substructure than for the other cases.

Similarly, Figure 4 plots the thermoelastic displacement phase shift for the same experimental parameters. It should be noted that the phase shift shown on this figure corresponds to the probe laser position coincident with the excitation laser position. Indeed, other results should be obtained if the probe laser position is shifted from the excitation laser position. Particularly, this shift usually results in a stronger phase shift (a best phase sensitivity) but also results in a weaker signal to noise ratio so that an optimal value has to be found. Moreover the higher is the modulation frequency, the higher is the phase shift.
But we have to take care that increasing the modulation frequency results in a smaller thermal penetration depth and also in a weaker signal to noise ratio.

Figure 4 shows that the thermoelastic displacement phase shift has a higher range for the $w=5 \mu \mathrm{m}$ case than for the $w=0.5 \mu \mathrm{m}$ case which is in agreement with the thermoelastic displacement magnitude analysis.

\section{Conclusion}

Finite element analysis is a useful tool for modeling thermoelastic fields. In the first part of this paper we have described a dynamic method based on FEA complex analysis for both thermal and thermoelastic fields. The second part then has shown that our FEA moduli enables to describe complicated samples (complex geometries, unhomogeneous and anisotropic physical properties). Thanks 

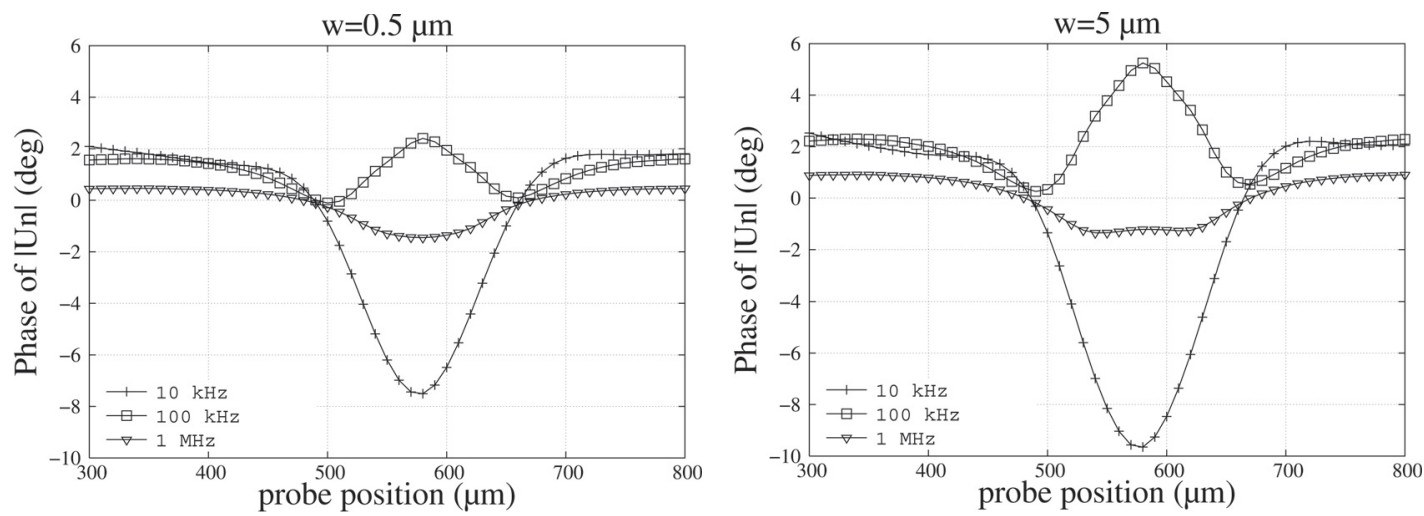

Fig. 4. Thermoelastic displacement phase shift.

to this FEA dynamic method we are able now to quantitatively analyze both photothermal and thermoelastic images. Consequently, this method will be dedicated to the analysis of thermal imaging system and more generally for addressing complex inverse problems specific to photothermal and thermoelastic microscopies. By coupling this FEA moduli with our experimental setup through optimization algorithms, we aim to make quantitative nondestructive evaluation.

\section{References}

1. D.P. Almond, P.M. Patel, Photothermal Science and Techniques (London, 1996)

2. S. Ballandras, Wilm, W. Daniau, A. Reinhardt, V. Laude, R. Armati. J. Appl. Phys. 034901 (2005)

3. B. Cavallier, Microscopies Photothermiques et Thermoelastiques Conventionnelles et à Sonde Locale: Theorie et Experimentation, in PhD Thesis, University of Franche-Comté, France, 2000

4. B. Cavallier, S. Ballandras, B. Cretin, P. Vairac. Sensors and Actuators A: Physical, 444 (2005)

5. B. Cavallier, S. Ballandras, B. Cretin, P. Vairac. J. Appl. Phys. 2006, 044509

6. B. Cretin, N. Daher, B. Cavallier, In Proceedings of SPIE Optical Inspection and Micromeasurements II (Munich, 1997), p. 466
7. J. Fivez, J. Thoen. J. Appl. Phys. 2225 (1996)

8. G. Goch, M. Reigl. J. Appl. Phys. 9084 (1996)

9. INRIA, MODULEF: un code modulaire d'elements finis, Rapports techniques, http://www-rocq.inria.fr (1984).

10. J.A. Jauregui, E. Welsch. Journal of Modern Optics, 2173 (1993)

11. M. Lax. J. Appl. Phys. 3919 (1977)

12. B.C. Li. J. Appl. Phys. 482 (1990)

13. A. Mandelis. J. Appl. Phys. 647 (1995)

14. A. Mandelis. J. Appl. Phys. 1811 (1999)

15. F.A. McDonald, Laser-Generated Ultrasound: Towards Realistic Modeling, In Proceedings of Photoacoustic and Photothermal Phenomena II, 262 (1990)

16. W. Nowacki, Thermoelasticity, 2nd edn. (Oxford, 1986)

17. H. Press, A. Teukolsky, T. Vetterling, P. Flannery, Numerical Recipes in Fortran 7r: The Art of Scientific Computing (Vol. 1 of Fortran Numerical Recipes) (2001)

18. M. Qian, Y. Pan, C. Bescond, M. Deschamps, B. Audoin. Progress in Natural Science, S378 (1996)

19. Yong-Feng Lu. Appl. Phys. Lett., 2482 (1992)

20. S. Zhou, P. Reynolds, R. Krause, T. Buma, M. O'Donnell, A. Hossack. IEEE Transactions on Ultrasonics, Ferroelectrics, and Frequency Control, 1178 (2004)

21. O.C. Zienkiewicz, The Finite Element Method 4th edn., (London, 1989) 